\title{
BurnCase 3D - Realistic Adaptation of 3-Dimensional Human Body Models
}

\author{
Johannes Dirnberger ${ }^{1}$, Michael Giretzlehner ${ }^{1}$, Thomas Luckeneder $^{1}$, Doris Siegl ${ }^{1}$, \\ Herbert L. Haller ${ }^{2}$, and Christian Rodemund ${ }^{2}$ \\ ${ }^{1}$ Upper Austrian Research, Dept. Medical Informatics, AT; \\ ${ }^{2}$ AUVA, Unfallkrankenhaus Linz \\ info@burncase.at
}

\begin{abstract}
This paper presents the results of the research project BurnCase in the field of realistic and anatomically correct deformations of 3D models of the human body. The project goal is to develop a software system named BurnCase $3 \mathrm{D}$, which supports and enhances the documentation and diagnosis of human burn injuries.

The medical treatment of burn victims strongly depends on size, depth, degree and location of the burnt skin. The size of the affected region is usually expressed as a percentage of the total body surface area (TBSA). Standardized 2D charts (e.g. Lund and Browder, Rule-Of-Nines, etc.) help to determine the percentage of the burnt surface area in relation to the total body surface area. However, body proportions highly influence the distribution of body surface area along the body [Livingston and Lee, 2000]. Thus, standard charts can only give rough approximations of the burnt surface area compared to the real size of an injury on a specific patient.

The software system BurnCase 3D will enhance this commonly applied 2D approximation process by introducing a 3D model of the patient's body. This 3D model provides a higher accordance to the real patient's surface area than any $2 \mathrm{D}$ chart does and allows determining the burnt surface areas more exactly. BurnCase 3D is based on an extendable library of currently 7 standard models representing different sex, age and body shape. In order to meet the physical constitution of the real patient, the best fitting model is chosen and has to be adapted according to the patient's height and weight. There exist several possibilities of adapting a 3D model to these parameters. This paper describes the three methods of body adaptations that are realized in the software system BurnCase 3D based on the thesis of Doris Siegl ([Siegl, 2003]).
\end{abstract}

\section{Introduction}

Human body shapes show an enormous variability. They are influenced by racial characteristics, alimentation habits and genetic predisposition. We can rely on our sensitivity to the familiar shape of human bodies to recognize correct body forms. Body measurement data, so-called anthropometric data [Flügel et al., 1986], defines the scope of possible proportions more exactly. 
The medical treatment of burn victims strongly depends on size, depth, degree and location of the burnt skin. The size of the affected region is usually expressed as a percentage of the total body surface area (TBSA). Standardized 2D charts (e.g. Lund and Browder, Rule-Of-Nines, etc.) help to determine the percentage of a specific body part in relation to the whole body surface. However, body proportions highly influence the distribution of body surface area along the body [Livingston and Lee, 2000]. Thus, standard charts are not sufficient for reliable surface estimations.

With the aid of a software tool, which uses a three-dimensional model representing the body measurements of the real patient, the burnt surface size can be determined more exactly and leads to enhanced medical treatment and shortened stay in hospital.

\section{Research Project BurnCase}

The goal of the research project BurnCase is to develop a software system, which supports and enhances the documentation and diagnosis of human burn injuries, thus alleviating the large variations among surgeons regarding approximation of size and depth of burn injuries. The problem of approximations of body surface areas is described in the introduction. Another problem in modern burn care is the large variety in quantity and quality of surveyed data among different burn units as well as the lack of mechanisms to exchange documented data. In order to address this problem, BurnCase 3D offers the possibility of comprehensive documentation of a burn case from admission until discharge.

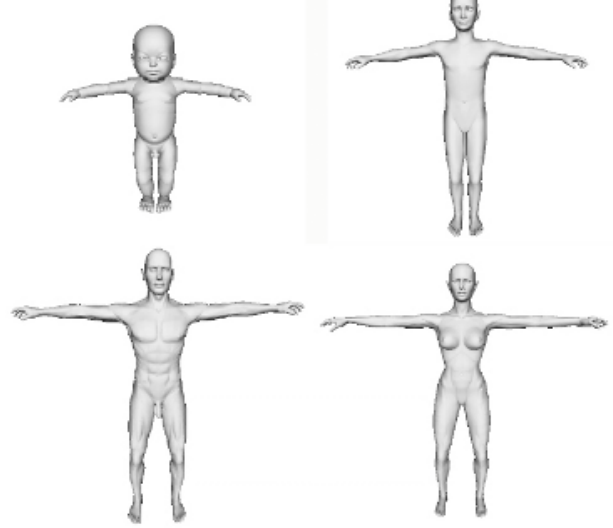

Fig. 1. Standard model library of BurnCase 3D

The strength of BurnCase 3D is the intuitive 3-dimensional graphical user interface that provides direct interaction with a virtual model of the patient's body. Thus, representations of injured regions can be transferred onto this model by using standard input devices like the mouse. Then, the diagnosis is enhanced by modern software technologies involving graphical interaction, complete database support and analysis methods. Accordingly one of the main goals is to provide a user interface which is practicable in a surgeon's daily use. 
BurnCase 3D is based on a library of various standard models representing different sexes, ages and body shapes. Figure 1 shows four examples of these standard models. These models have been generated by modern game development software and allow a selection accuracy of one $\mathrm{cm}^{2}$.

\section{Internal Model Representation}

The triangle mesh that represents the patient's body in the virtual environment of the BurnCase 3D software system is embedded in an object oriented data structure. The model entity encapsulates the whole model information containing the following types of information:

- Sex and age range

- Axis assignment (which axis defines height, width, breadth)

- Array of 3-dimensional points of the model mesh (vertices)

- Array of triangles that build up the model surface (polygons)

Body surface adaptation algorithms are strongly dependent on the information which axis of the Cartesian coordinate system does represent body height, which one body width and which one body depth. These algorithms use the axis information and transform the 3-dimensional model points. Since the human body does not grow or shrink uniformly on gain or loss of weight these algorithms are non-trivial and have to consider information of location and associated body region's growth behavior.

Thus, triangles do not only know the 3 edge points (addressed by vertex indices) but they contain the following additional information which is necessary for adaptation as well as for visualization and automated medical encoding respectively:

- Anatomical body region (e.g. head, face, thorax, pelvis, etc.)

- Characteristic of injury (e.g. burn degree, necrosectomy, etc.)

- Color for visualization

- Stipple for visualization

- Normal vector for light calculation

- Surface area of the triangle

- Length of longest edge

- Link to neighboring triangles

The normal vector of each triangle gives the direction which side of the triangle lies interior and which one lies exterior regarding the 3-dimensional human model.

\section{Realistic Model Adaptation}

The different standard models already provide an accurate approach to the real patient's body shape. However, it is necessary to adapt the surface of these standard 
models to fit the patient's height and weight since all the models are available for one standard height only: $170 \mathrm{~cm}$ for adults, $150 \mathrm{~cm}$ for children and $60 \mathrm{~cm}$ for infants. After choosing an appropriate standard model for the patient the surgeon specifies height and weight of the patient. BurnCase $3 \mathrm{D}$ adapts the chosen standard model according to the entered body measurements. This adaptation procedure is described in the following chapters.

\subsection{Body Height Adaptation}

First of all, the model mesh has to be adapted in terms of body height. This is achieved by simply stretching or shrinking the mesh along the longitudinal body axis (vertical axis along the spine). This is done by simply multiplying every vertex by a factor. Since the model surface area growth is not linearly dependent on this multiplication factor the factor has to be adapted by linear error regression iteration. The factor is thereby calculated as follows:

$$
\text { factor }_{\text {height }}=\left\{\begin{array}{cl}
\frac{h_{\text {dest }}}{h_{\text {current }}}, & \frac{h_{\text {dest }}}{h_{\text {current }}} \geq 2 \\
2-\frac{h_{\text {dest }}}{h_{\text {current }}}, & \text { else }
\end{array}\right.
$$

This height adaptation is, however, not sufficient for realistic model adaptation. A patient of $180 \mathrm{~cm}$ and $70 \mathrm{~kg}$ has a completely different body shape than a patient of the same height and $120 \mathrm{~kg}$. Thus, the body surface has to be adapted to the patient's weight too.

\subsection{Body Weight Adaptation}

In order to correctly adapt the model according to the patient's weight the average body surface area (BSA) is calculated based on several formulas, which approximate the BSA according to age, sex, weight and height based on statistical data. The model adaptation is done by expanding the surface along the normal vectors of the mesh polygons, until the approximated destination surface area is reached. This adaptation must not change the model proportions between corpus and extremities and it takes the varying growing behaviors of different body regions into account.

The following standard formulas for calculating the BSA are integrated into the BurnCase 3D Core system and can be chosen by the surgeon alternatively.

Mosteller [Mosteller, 1987]

$\mathrm{A}_{\mathrm{BSA}} \ldots$... Body Surface Area $\left[\mathrm{m}^{2}\right] \quad A_{B S A}=\left(\frac{H_{B} * W_{B}}{3600}\right)^{\frac{1}{2}}$

$\mathrm{H}_{\mathrm{B}} \quad \ldots$ Body Height $[\mathrm{cm}]$

$\mathrm{W}_{\mathrm{B}} \quad \ldots$ Body Weight $[\mathrm{kg}]$ 
DuBois \& DuBois [DuBois\&DuBois, 1916] $\quad A_{B S A}=0.20247 \cdot H_{B}{ }^{0.725} \cdot W_{B}^{0.425}$

$\mathrm{A}_{\mathrm{BSA}} \ldots$... Body Surface Area $\left[\mathrm{m}^{2}\right]$

$\mathrm{H}_{\mathrm{B}} \quad \ldots$ Body Height $[\mathrm{m}]$

$\mathrm{W}_{\mathrm{B}} \quad \ldots$ Body Weight $[\mathrm{kg}]$

Haycock [Haycock, 1978]

$\mathrm{A}_{\mathrm{BSA}} \ldots$... Body Surface Area $\left[\mathrm{m}^{2}\right]$

$\mathrm{H}_{\mathrm{B}} \quad \ldots$ Body Height $[\mathrm{cm}]$

$\mathrm{W}_{\mathrm{B}} \quad \ldots$ Body Weight $[\mathrm{kg}]$

Gehan \& George [Gehan\&George, 1970] $\quad A_{B S A}=0.0235 \cdot H_{B}^{0.42246} \cdot W_{B}^{0.51456}$

$\mathrm{A}_{\mathrm{BSA}} \ldots$ Body Surface Area $\left[\mathrm{m}^{2}\right]$

$\mathrm{H}_{\mathrm{B}} \quad \ldots$ Body Height $[\mathrm{cm}]$

$\mathrm{W}_{\mathrm{B}} \quad \ldots$ Body Weight $[\mathrm{kg}]$

Boyd [Boyd, 1930]

$\begin{array}{lll}\mathrm{A}_{\mathrm{BSA}} & \ldots \text { Body Surface Area }\left[\mathrm{m}^{2}\right] \\ \mathrm{H}_{\mathrm{B}} & \ldots \text { Body Height }[\mathrm{cm}] \\ \mathrm{W}_{\mathrm{B}} & \ldots \text { Body Weight }[\mathrm{g}]\end{array}$

$A_{B S A}=0.0003207 \cdot H_{B}^{0.3} \cdot W_{B}^{\left(0.7258-\left(0.0188 \cdot \log \left(W_{B}\right)\right.\right.}$

Lam \& Leung [Lam\&Leung, 1988]
$\mathrm{A}_{\mathrm{BSA}} \ldots$... Body Surface Area $\left[\mathrm{m}^{2}\right]$
$\mathrm{H}_{\mathrm{L}} \quad \ldots$ Body Height $[\mathrm{cm}]$
$\mathrm{W}_{\mathrm{L}} \quad \ldots$ Body Weight $[\mathrm{kg}]$

$$
A_{B S A}=0.024265 \cdot H_{B}^{0.3964} \cdot W_{B}^{0.5378}
$$

These formulas give good approximations of the BSA for adults in western populations. BurnCase 3D calculates the approximated surface area according to one of these formulas based on the patient's height and weight. After the standard model has been uniformly stretched along the longitudinal axis to fit the body height BurnCase 3D starts the expansion algorithm until the model fits the calculated BSA. Therefore, every triangle's edge vertices are translated along the direction of the exterior normal vector of each vertex.

Let the set of $\mathrm{m}$ adjacent triangles $\mathrm{T}$ for $\mathrm{V}_{\mathrm{i}}$ (triangles that contain $\mathrm{V}_{\mathrm{i}}$ ) be defined as

$$
T\left(V_{i}\right)=\left\{t_{1}, t_{2}, \ldots t_{m} \mid V_{i} \in t_{i}\right\}
$$

Each triangle $\mathrm{t}_{\mathrm{i}}$ in $\mathrm{T}$ defines a normal vector $\bar{N}$ perpendicular to the triangle's plane. Let the three vertices of $t_{i}$ be $V_{1}, V_{2}$, and $V_{3}$ then the triangle's normal vector is defined as

$$
\bar{N}\left(t_{i}\right)=\overrightarrow{V_{1} V_{2}} \times \overrightarrow{V_{2} V_{3}}
$$

Consequently, the normal vector $\mathrm{N}$ of vertex $\mathrm{V}_{\mathrm{i}}$ is the average vector of all adjacent triangle's normal vectors and can be described as 


$$
N\left(V_{i}\right)=\left(\sum_{t_{i} \in T\left(V_{i}\right)} \frac{\bar{N}\left(t_{i}\right)}{\left|\bar{N}\left(t_{i}\right)\right|}\right) \frac{1}{m}
$$

Where $\mathrm{m}$ is the size of $\mathrm{T}\left(\mathrm{V}_{\mathrm{i}}\right)$. The normal vector $\bar{N}$ has to be normalized (division by vector length) in order to receive the correct vertex normal. A linear iteration algorithm computes the factor of translation for every iteration step as

$$
\text { factor }_{B S A}= \begin{cases}\frac{B S A_{\text {dest }}}{B S A_{\text {current }}}-1, & \frac{B S A_{\text {current }}}{B S A_{\text {dest }}} \geq 2 \\ 1-\frac{B S A_{\text {current }}}{B S A_{\text {dest }}}, & \text { else }\end{cases}
$$

This factor ${ }_{\mathrm{BSA}}$ is multiplied by the normal vector of each vertex. The resulting product vector is added to the vertex by vector addition which causes the vertex to move exterior or interior along the direction of its normal vector. The direction is dependent on whether the destination BSA is greater (causing a positive factor ${ }_{\mathrm{BSA}}$ ) or smaller than the current BSA (causing a negative factor ${ }_{\mathrm{BSA}}$ ).

This method converges in most cases, but for extreme values of body height and body weight the iteration may not converge. Thus, BurnCase 3D limits this iterative adaptation to a maximum of 300 iteration steps. The average amount of iterations until the destination height and BSA is reached lies between 4 and 20 steps. For performance reasons the body height adaptation and the body weight adaptation are combined in one iteration procedure. Consequently, less than 20 iterations are needed to adapt both height and BSA of a standard model to a real patient.

\subsection{Body Region Growth Behaviors}

The adaptation of the model according to body height and weight is described in chapter 4.2. However, the calculation of factor $_{\mathrm{BSA}}$ does not take different growth behaviors of different body regions into account. This leads to inappropriate and unrealistic results for patients whose body shapes differ from the body shape of the chosen standard model. In order to avoid these failures each body region additionally defines a growth regression rate. This growth regression rate has a domain of $[1$, 1000] where 1 means default growth behavior (e.g. chest, stomach, etc.) and 1000 means maximum growth regression (e.g. ear, palpebra).

Thus, a realistic vertex translation along the vertex normal can be applied as follows:

$$
\vec{v}_{i}=\vec{v}_{i}+\left(\vec{n}_{i} \frac{\text { factor }_{B S A}}{\text { rate }_{\text {regression }}}\right)
$$

Where $\vec{v}$ is the vertex and $\vec{n}$ is the normalized normal vector of this vertex. Without such a growth regulation the model would be deformed unproportionally as depicted in figure 2. Application of equation (12) performs much better and leads to more realistic results as shown in figure 3 . 


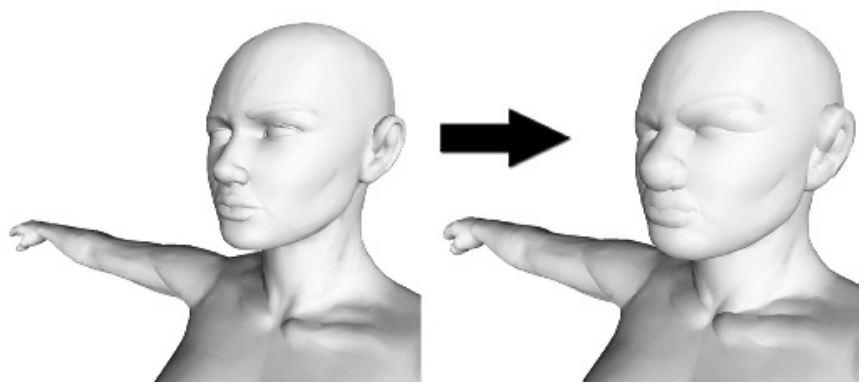

Fig. 2. Unproportional body deformation of the face as consequence of equal growth behaviors of body regions (left: $160 \mathrm{~cm}, 55 \mathrm{~kg}$; right: $160 \mathrm{~cm}, 90 \mathrm{~kg}$ )

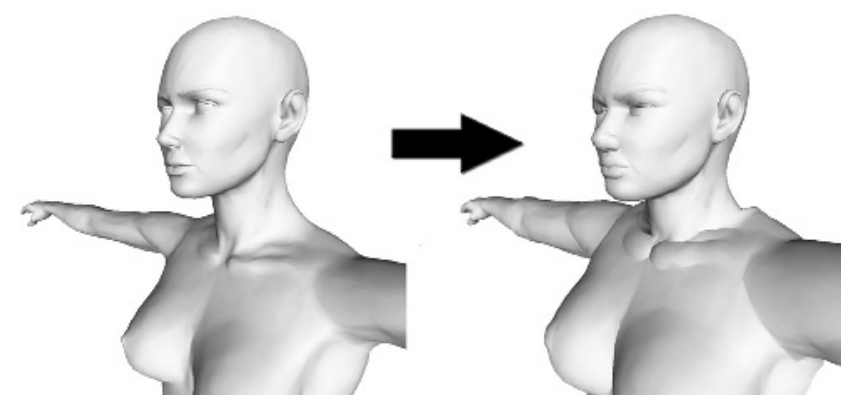

Fig. 3. More realistic body adaptation, taking different growth behaviors of certain body regions into account (left: $160 \mathrm{~cm}, 55 \mathrm{~kg}$; right: $160 \mathrm{~cm}, 90 \mathrm{~kg}$ )

\section{Conclusion}

The software system BurnCase 3D is already in use in several burn units in Austria and Germany. First results showed up a high reliability of the calculated burnt surface areas on the adapted standard models. In particular, the system is used to determine the surface area of remaining healthy skin that could be used for skin transplantation. The results were verified after the surgery and showed an accordance of only a few square centimeters.

Although the adaptation results are very promising, there are still several drawbacks that have to be overcome. Extreme body shapes cause the adaptation algorithm to build rough edges on the border line between two body regions with different growth behaviors. This can be observed e.g. on the shoulder blade in Figure 3 since shoulders and thorax grow much faster than the neck, resulting in unrealistic edges. [Siegl, 2003] shows that it is possible to refine the deformation results by application of several different smoothing algorithms like iterative smoothing, curve smoothing (sigmoid smoothing) and graded smoothing. These algorithms can smooth the borders between body regions with different growth behaviors. Furthermore, it is suggested to define different types of deformation each defining a specific set of growth 
parameters. Thus, the typical male type of deformation bulges more stomach and thighs whereas the typical female deformation type applies major growth on buttocks and legs.

The adaptation of the model is performed based on the size of the model's body surface area. As long as the model's BSA does not approximately match a calculated $\mathrm{BSA}$, the deformation algorithm is performed. Due to the fact that these BSA formulas just give approximations which sometimes diverge immensely from a real person's BSA, another approach should be taken into consideration. Not the calculated BSA, but measurements entered by the surgeon should act as termination criterion for the model deformation algorithm. If the model measurements became of greater importance for the deformation process also the measurement methods would have to become more flexible. At present, the measurement methods are only used in order to evaluate the results of the model deformation. However, in clinical practice it is often very difficult to measure parameters like arm perimeter, thigh perimeter, chest perimeter, and many more.

Since July 2003 a first version of the software system is being tested in 3 hospitals in Austria, one hospital in Germany followed February 2004. The results are promising and show that the improved estimation process of injured surface area will have influences in modern burn treatment especially in the application of formulas for fluid donation and planning of skin transplantation. Nevertheless, further investigations on the adequacy and accuracy of the software results are necessary.

\section{References}

[Boyd, 1930] Boyd E: Experimental error inherent in measuring growing human body, Am J Physiol 1930; 13:389-432.

[DuBois\&DuBois, 1916] DuBois D and DuBois EF: A formula to estimate the approximate surface area if height and weight be known. Arch Intern Med 1916; 17:863-71.

[Flügel et al., 1986] Flügel B, Greil H, Sommer K: Anthropologischer Atlas. Edition Wötzel, 1986.

[Gehan\&George, 1970] Gehan EA, George SL: Estimation of human surface area from height and weight. Cancer Chemother Rep part 1 1970; 54:225-35.

[Haycock, 1978] Haycock GB, Schwarta GJ, Wistosky DH: Geometric method for measuring body surface area: A height-weight formula validated in infants, children, and adults. $\mathrm{J}$ Pediatr 1978; 93:62-6

[Lam\&Leung, 1988] Lam TK, Leung DT: More on simplified calculation of body-surface area, N Engl J Med 1988 Apr 28; 318(17):1130, (letter)

[Livingston and Lee, 2000] Livingston EH, Lee S: Percentage of burned body surface area determination in obese and nonobese patients. J Surg Res. 2000, 91(2):106-10.

[Mosteller, 1987] Mosteller RD: Simplified calculation of body surface area. N Engl J Med 1987; 317:1098

[Siegl, 2003] Siegl D: Virtual Human Body Deformation for Medical Applications. Diploma Thesis, Polytechnic University of Hagenberg, Software Engineering for Medical Purposes, 2003. 